BULL. AUSTRAL. MATH. SOC.

VOL. II (1974), 443-454.

\title{
On equidistant sets in normed linear spaces
}

\section{B.B. Panda and O.P. Kapoor}

\begin{abstract}
In this note some results concerning the equidistant set $E(-x, x)$ and the kernel $M^{\theta}$ of the metric projection $P_{M}$, where $M$ is a Chebyshev subspace of a normed linear space $X$, have been obtained. In particular, when $X=2^{p} \quad(1<p<\infty)$, it has been proved that every equidistant set is closed in the $b w$-topology of the space. In $c_{0}$ no equidistant set has this property.
\end{abstract}

\section{Introduction}

Let $X$ be a real normed linear space. For any two distinct points $x$ and $y$ of $X$, let $E(x, y)$ denote the equidistant set from $x$ and $y$; that is, the set of points $p$ in $X$ for which $\|p-x\|=\|p-y\|$. Such sets were introduced by Kalisch and Straus in [6] in connection with their study of "determining" sets in Banach spaces. In an inner-product space every set $E(x, y)$ is a closed hyperplane, but in general it may not be even weakly closed. Not much is known about spaces other than inner-product and finite dimensional spaces in which sets $E(x, y)$ are weakly or weakly sequentially closed. The purpose of this paper is to make an attempt in that direction.

In the first section we shall study a few geometrical and topological properties of the set $E(x, y)$. For example, in Theorem 1.2 we prove that, if $E(x, y)$ is convex, then it is a hyperplane and as a consequence,

Received 31 August 1974. 
the convexity of all sets $E(x, y)$ implies that the space $X$ is an innerproduct space. The connection between the structural properties of the set $E(-x, x)$ and those of the kernel $M^{\theta}$ of the metric projection $P_{M}$ where $M$ is the linear span of the point $x$, is then exhibited in Theorem 1.4 and Lemma 1.5. These results are closely related to the recent works of Holmes and Kripke [4], Kottman and Lin [8], and Holmes [3].

In the second section of this paper we show that $\imath^{p}$ spaces $(I<p<\infty)$ have the property that all sets $E(x, y)$ are closed in the bounded weak topology. Thus these spaces satisfy the $P_{2}$-property (see Klee [7], p. 298). In contrast to $l^{p}$-spaces, we find that in $c_{0}$, $E(x, y)$ is not even weakly sequentially closed for any $x$ and $y \in c_{0}$.

\section{Some properties of the equidistant set $E(-x, x)$}

We begin by recalling some notations and definitions. Let $X$ be a normed linear space over the real numbers $R$, with $\theta$ as its zero element. Let $x \in X$ and $K \subset X$. A point $y \in K$ is called a nearest point of $x$ in $K$, if $\|x-y\| \leq\|x-z\|$ for every $z \in K$. A set $K \subset X$ is said to be proximal (respectively, Chebyshev) if for each point $x \in X$, there exists a (respectively, a unique) nearest point of $x$ in $K$. Let $M$ be a Chebyshev linear subspace of $X$. The metric projection supported by $M$ will be denoted by $P_{M}$. It is known [3,p. 160] that $P_{M}$ induces a direct sum decomposition of $X$. Namely, every $x \in X$ can be written uniquely as $x=m+y$ where $m \in M$ and $y \in M^{\theta}$, where $M^{\theta}=\left\{x \in X: P_{M}(x)=\theta\right\} . M^{\theta}$ is called the kernel of $P_{M}$.

. For $x \neq \theta$ in $X$, let $E(-x, x)$ denote the equidistant set from $x$ and $-x$; that is, the set of points $y \in X$ such that $\|y-x\|=\|y+x\|$. Observe that each equidistant set is closed. If $x$ and $y \in X$ and $\|x-y\|=\|x+y\|$ we say that $x$ is orthogonal to $y$ and write $x \perp y$. Thus $E(-x, x)$ is then the set of all vectors in $X$ which are orthogonal to $x$. This concept of orthogonality is named the isosceles orthogonality and has been studied by James in [5]. We shall need the following result from [5]. For each pair of linearly independent vectors $x$ and $y$ in 
$X$, there exists a number $t \in R$ such that $t x+y \perp x$. By a cone in $X$, we shall mean a set $K$ such that $x \in K \Rightarrow t x \in K$, for every nonnegative number $t$. With these preliminaries we pass on to the study of some geometrical and topological properties of the set $E(-x, x)$.

LEMMA 1.1. Let $x$ be any point of a two dimensional normed linear space $X$. If $E(-x, x)$ is convex then it must be a line through the origin.

Proof. Let $E(-x, x)$ be convex and $z \neq \theta \in E(-x, x)$. By the result of James for isosceles orthogonality such a point $z$ exists. We shall show that $E(-x, x)=[z]$, the one-dimensional subspace spanned by $\boldsymbol{z}$. First, since $E(-x, x)$ is symmetric about the origin, the convexity implies that $\{t z:|t| \leq 1\} \subset E(-x, x)$. If $y \in E(-x, x)$ is linearly independent from $z$, then either $y$ and $z$ or $-y$ and $z$ are separated by the line $[x]$. Since $y \in E(-x, x)$ implies $-y \in E(-x, x)$, we assume that the former holds. Then the line segment joining. $y$ and $z$ is contained in $E(-x, x)$; but since this line intersects [x] at a point other than the origin it cannot be a point of $E(-x, x)$. Hence there is a contradiction.

Now we show that $E(-x, x)$ is unbounded. Let $z \in E(-x, x)$ and $\lambda>1$ be arbitrary. Then again by James' result there exists a $t$ in $R$ such that $\lambda z+t x \in E(-x, x)$. This implies that $z$ and $\lambda z+t x$ must be linearly dependent. This is possible only if $t=0$. Hence the result is proved.

THEOREM 1.2. Let $x \neq \theta$ be any point of a normed linear space $X$. If $E(-x, x)$ is convex then it must be a proximal subspace of codimension one.

Proof. Let $E(-x, x)$ be convex and $z$ be any point of $X$ outside [x]. Then $E(-x, x) \cap[x, z]$ is convex and by Lemma 1.1 , it must be a line. Thus if $z \in E(-x, x)$, then $[z] \subset E(-x, x)$. Consequently, $E(-x, x)$ is a convex cone symmetric about the origin. Hence it is a subspace. Now let $u \in X$. Then either $u=\lambda x$ or, by James' result, $u+\lambda x=z \in E(-x, x)$ for some $\lambda$. Thus $E(-x, x)$ and $x$ together span $X$. Therefore $E(-x, x)$ is of codimension 1 . Since every equidistant set is closed it follows that $E(-x, x)$ is a closed subspace.

Now let $h \in E(-x, x)$. Then $\|x-h\|=\|x+h\|$ and hence $\theta$ is a 
nearest point of $x$ in $E(-x, x)$. If $\alpha \in R$, then

$$
\|\alpha x-h\|=|\alpha|\left\|x-\alpha^{-1} h\right\|=|\alpha|\left\|\mid x+\alpha^{-1} h\right\|=\|\alpha x+h\|
$$

for all $h$ in $E(-x, x)$ and hence $\theta$ is also a nearest point in $E(-x, x)$ to $\alpha x$. As any $w \in X$ has a representation $w=\alpha x+h$, where $h \in E(-x, x)$, we have

$$
\|w-h\|=\|\alpha x\| \leq\|\alpha x-z\|, z \in E(-x, x),
$$

which implies

$$
\|w-h\| \leq\|w-z-h\|, \quad z \in E(-x, x) .
$$

But $E(-x, x)$ being a subspace, $z+h \in E(-x, x)$ and hence $\|w-h\| \leq\|w-v\|$ for every $v$ in $E(-x, x)$. Thus every $w$ in $X$ has a nearest point in $E(-x, x)$.

As a consequence of the above theorem we have, under weaker assumptions, the following characterization of inner-product spaces [ $]$, Theorem 5.4].

COROLLARY 1.3. Let $X$ be a normed linear space. If $E(-x, x)$ is convex for each $x \in X$, then $X$ must be an inner-product space.

Proof. Immediate from the above theorem and Day's result.

In the sequel, $M^{\theta}$ denotes the kernel of the metric projection $P_{M}$, where $M$ is a Chebyshev subspace. We then have the following theorem.

THEOREM 1.4. Let $M$ be the one dimensional span [x] of $x$ in a normed linear space $X$. Let $M$ be Chebyshev. Then the following hold:

$$
\begin{gathered}
M^{\theta} \subset E(-x, x) \Rightarrow M^{\theta}=E(-x, x) ; \\
E(-x, x) \text { is a cone } \Rightarrow M^{\theta}=E(-x, x) .
\end{gathered}
$$

We need the following result in its proof.

LEMMA 1.5 [8, Lemma 1]. If $x \in X$ and $M=[x]$ is Chebyshev, then

$$
P_{M}(E(-x, x)) \subset\{t x:-1 \leq t \leq 1\} \text {. }
$$

Proof of Theorem $1.4\left(1^{\circ}\right)$. If $u \in E(-x, x)$ then $P_{M}(u)=\alpha x$ with $|\alpha| \leq 1$. Since $\|u-x\|=\|u+x\|$ and $u$ has a unique nearest point in 
$[x],|\alpha| \neq 1$. We can write $u=u_{\theta}+\alpha x$ where $u_{\theta} \in M^{\theta}$ and, since $\lambda u_{\theta} \in M^{\theta} \subset E(-x, x)$ for all $\lambda \in R$, we have $u_{\theta} \perp \mu x$ for all $\mu \in R$; that is,

$$
\left\|u_{\theta}-\mu x\right\|=\left\|u_{\theta}+\mu x\right\|, \quad \mu \in R
$$

In particular, with $\mu=1-\alpha$, we have

$$
\begin{aligned}
\|u+(1-2 \alpha) x\| & =\left\|u_{\theta}+\alpha x+(\mu-\alpha) x\right\|=\left\|u_{\theta}+\mu x\right\| \\
& =\left\|u_{\theta}-\mu x\right\|=\|u-x\|=\|u+x\|=r, \text { (say). }
\end{aligned}
$$

So the sphere centred at $u$ and radius $r$ meets $M$ in at least three points: $x,+x$, and $(-1+2 \alpha) x$, which is impossible unless $1-2 \alpha= \pm 1$. Thus $\alpha=0$ or $\alpha=+1$ and the latter, we saw above, is also impossible. Therefore $\alpha=0$ and $u \in M^{\theta}$.

$\left(2^{\circ}\right)$. Let $u \in M^{\theta}$. Then there exists a number $t$ such that $u-t x \in E(-x, x)$. Since $P_{M}(E(-x, x)) \subset\{a x:|a| \leq 1\}$, we have $|t| \leq 1$. Because $E(-x, x)$ is a cone, $u-t x \in E(-x, x)$ implies $\lambda u-\lambda t x \in E(-x, x)$ for arbitrary $\lambda$ in $R$. But $\lambda u \in M^{\theta}, \forall \lambda \in R$, and hence we must have $|\lambda t| \leq 1$. This is possible only when $t=0$. Hence $M^{\theta} \subset E(-x, x)$ and, by $\left(I^{\circ}\right)$ above, $M^{\theta}=E(-x, x)$.

However, $M^{\theta}$ is a subspace does not imply that $E(-x, x)$ is also a subspace. In fact Kottman and Lin [8] have given an example where $M^{\theta}$ is a closed hyperplane, but $E(-x, x)$ is not even weakly sequentially closed.

In the following we see the relation between $M^{\theta}$ and $E(-x, x)$ as regards weak topology, where $M=[x]$ is given to be Chebyshev. We give a simple proof of a result in [8].

THEOREM 1.6. Let $M=[x]$ be a Chebyshev subspace of a normed linear space $X$. Then $M^{\theta}$ is weakly (bounded weakly, or weakly sequentially) closed if $E(-x, x)$ is weakly (bounded weakly, or weakly sequentially) closed.

Proof. We consider the case when $E(-x, x)$ is weakly closed, the 
other cases being similar. Let $\left\{u_{\alpha}\right\} \subset M^{\theta}$ be a net which converges weakly to $u \in X$. Suppose that $u \neq M^{\theta}$; then taking $2 z=P_{M}(u)$ we can find a net $\left\{t_{\alpha}\right\}$ of real numbers such that $u_{\alpha}-t_{\alpha} z \in E(-z, z)$ and $\left|t_{\alpha}\right| \leq 1$. If $t_{0}$ is a cluster point of the net $\left\{t_{\alpha}\right\}$, then $\left|t_{0}\right| \leq 1$, and since $E(-z, z)$ is weakly closed, being a scalar multiple of $E(-x, x)$, $u-t_{0} z \in E(-z, z)$. Therefore, $P_{M}\left(u-t_{0} z\right)=2 z-t_{0} z \in\{t z:|t| \leq 1\}$. This means $1 \leq t_{0} \leq 3$ and hence $t_{0}=1$. It follows then that $u-z \in E(-z, z)$; that is, $\|u-\theta\|=\|u-2 z\|=\left\|u-P_{M}(u)\right\|$, and this contradicts the Chebyshev property of $M$. This proves the result.

In the following we consider a structural property of the set $E(-x, x)$.

THEOREM 1.7. Let $E(-x, x)$ be a convex subset of a normed linear space $X$ with $\|x\|=1$. Then $E(-x, x)$ is Chebyshev if and only if $x$ is an extreme point of the unit ball of $X$.

Proof. Let $E(-x, x)$ be a Chebyshev set. It will be actually a subspace because of Theorem 1.2. If $x$ is not an extreme point of the unit ball of $X$, then there exists a pair of points $x_{1}$ and $x_{2}$ in the unit sphere $S=\{z \in X:\|z\|=1\}$ such that $x=\frac{1}{2}\left(x_{1}+x_{2}\right)$ and $I=\left\{t x_{1}+(1-t) x_{2}: 0 \leq t \leq 1\right\}$ is contained in $S$. Now

$$
\left\|x_{1}-x-x\right\|=\left\|x_{2}\right\|=1=\left\|x_{1}\right\|=\left\|x_{1}-x+x\right\|
$$

and hence $x_{1}-x \in E(-x, x)$. Similarly $x_{2}-x \in E(-x, x)$. Thus $x_{1}, x_{2} \in E(\theta, 2 x)$ and since $E(-x, x)$ is a subspace, $I \subset E(\theta, 2 x)$. As $E(-x, x)$ is Chebyshev and $h \in E(-x, x)$ implies that $\|x-h\|=\|x+h\|$, we must have

$$
1=\|x\|=\inf \{\|x-h\|: h \in E(-x, x)\} .
$$

Hence the origin is the nearest point of $x$ in $E(-x, x)$. This in turn implies that the origin has the nearest point $x$ in $E(\theta, 2 x)$. But $x \in I$ and every point of $I$ has norm 1 . This contradicts the Chebyshev property of $E(\theta, 2 x)$. 
Conversely, it is easy to see that if $x$ is an extreme point of the unit ball, then $\theta$ is the unique nearest point in $E(-x, x)$ to $\lambda x$, $\lambda \in R$. Hence if $u=z+\lambda x$, and $z \in E(-x, x)$, then $z$ is the unique nearest point to $u$. Therefore $E(-x, x)$ is Chebyshev.

In the following we illustrate Theorem 1.7 by two examples.

EXAMPLE 1.8. Take $X=R^{2}$ with the sup norm, $x=(1,1)$ and $z=(-1,1)$. It is easy to see that $E(-x, x)=[z]$ and $E(-z, z)=[x]$ are Chebyshev subspaces, and $x$ and $z$ are extreme points of the unit ball of $X$.

EXAMPLE 1.9. Let $X=i^{1}$ and let $e_{i}$ be the vector with 1 at the ith place and zero otherwise. Then $E\left(-e_{i}, e_{i}\right)=\left\{z \in l^{l}: z(i)=0\right\}$ is a closed hyperplane. If $u \in l^{l}$, then the unique nearest point to $u$ in $E\left(-e_{i}, e_{i}\right)$ is $z$, where $z(j)=\left(1-\delta_{i j}\right) u(j), \delta_{i j}$ being the Kronecker delta. Thus the set $E\left(-e_{i}, e_{i}\right)$ is Chebysher. Clearly $e_{i}$ is an extreme point of the unit ball of $l^{l}$. Also, if we write $M_{i}=\left|e_{i}\right|$, then $M_{i}^{\theta}=E\left(-e_{i}, e_{i}\right)$.

\section{Nature of equidistant sets in $\imath^{P}$ spaces}

Let $X$ be a normed linear space and let $E(x, y)$ be the equidistant set from $x$ and $y \in X$. The space $X$ is said to have

(1) property $P_{1}$ if for all $x, y \in X, E(x, y)$ is weakly closed,

(2) property $P_{2}$ if for each $x \in X$ with $\|x\|=1$, there exists $\varepsilon_{x}>0$ such that whenever $y$ and $z$ are distinct points of the set $x+\varepsilon_{x} U$, then the intersection $E(y, x) \cap\left(\varepsilon_{x} U\right)$ is weakly closed, $U$ denoting the unit cell of $X$.

That there is a connection between properties $P_{1}$ and $P_{2}$ and the continuity behaviour of metric projections onto Chebyshev sets is indicated by a result of Klee [7, Proposition 2.5]. Not much is known about spaces having the property $P_{1}$. Apart from the finite dimensional and inner- 
product spaces, no other example of spaces possessing the property $P_{1}$ has appeared in the literature. In the following we shall show that each equidistant set in an $Z^{p}$ space $(1<p<\infty)$ is closed in the bounded weak topology. It is easy to see that we need only consider equidistant sets of the form $E(-x, x)$. We start by proving a simple inequality.

LEMMA 2.1. Let $p \geq 1$ and $y$ and $z$ be any two complex numbers. Then the following inequality holds:

$$
|| y+\left.z\right|^{p}-|y-z|^{p} \mid \leq z^{p} p\left(\left|y^{p-1} z\right|+|z|^{p}\right) \text {. }
$$

Proof. Using the triangle inequality we see that we need only prove

$$
(|y|+|z|)^{p}-|| y|-| z||^{p} \leq 2^{p} p\left(\left|y^{p-1} z\right|+|z|^{p}\right) \text {. }
$$

The result then follows from the following simple inequality, which can be proved by using elementary methods of differential calculus:

$$
(1+x)^{p}-(1-x)^{p} \leq 2^{p} p\left(x+x^{p}\right), 0 \leq x \leq 1 .
$$

We next prove a variant of Lebesgue's Dominated Convergence Theorem for $l^{l}$. This will be used to prove the main result of this section.

THEOREM 2.2. Let $\left\{\Phi_{\alpha}, D\right\}$ be a net in $l^{l}$ converging pointwise to $\phi$. If there exists a net $\left\{f_{\alpha}, D\right\}$ in $l^{l}$ which converges in norm to an element $f$ and if $\left|\phi_{\alpha}\right| \leq f_{\alpha}$ for every $\alpha \in D$, then $\phi \in \eta^{1}$ and $\sum_{i=1}^{\infty} \phi_{\alpha}(i) \rightarrow \sum_{i=1}^{\infty} \phi(i)$

Proof. Clearly $\phi \in Z^{1}$. The rest then follows from the following inequality:

$$
\left|\sum_{i=1}^{\infty} \phi_{\alpha}(i)-\sum_{i=1}^{\infty} \phi(i)\right| \leq\left|\sum_{i=1}^{i_{0}} \phi_{\alpha}(i)-\sum_{i=1}^{i_{0}} \phi(i)\right|+\left\|f_{\alpha}-f\right\|+2 \sum_{i_{0}+1}^{\infty} f(i) .
$$

REMARK 2.3. Taking

$$
\phi_{n}=f_{n}=e_{n} / n \text { where } e_{i}(j)=\delta_{i j},
$$


and observing that $\left\{\phi_{n}\right\}$ is not dominated by a single $f \in l^{l}$, we see that Theorem 2.2.could be applied in situations in which Lebesgue's Dominated Convergence Theorem does not help.

THEOREM 2.4. Let $x$ be any point of $2^{P} \quad(1<p<\infty)$. Then $E(-x, x)$ is closed in the bounded weak topology of the space.

Proof. Let $\left\{u_{\alpha}, D\right\}$ be a bounded net in $E(-x, x)$ converging weakly to $u$. Then

$$
\left\|u_{\alpha}-x\right\|=\left\|u_{\alpha}+x\right\| \text { for all } \alpha \in D \text {; }
$$

that is,

$$
\sum_{i=1}^{\infty}|| u_{\alpha}(i)-\left.x(i)\right|^{p}-\left|u_{\alpha}(i)+x(i)\right|^{p} \mid=0 .
$$

Let

$$
\begin{aligned}
z_{\alpha}(i) & =\left|u_{\alpha}(i)-x(i)\right|^{p}-\left|u_{\alpha}(i)+x(i)\right|^{p}, \\
z(i) & =|u(i)-x(i)|^{p}-|u(i)+x(i)|^{p}, \\
w_{\alpha}(i) & =2^{p} p\left[\left|u_{\alpha}^{p-1}(i) x(i)\right|+|x(i)|^{p}\right], \\
w(i) & =2^{p} p\left[\left|u^{p-1}(i) x(i)\right|+|x(i)|^{p}\right], \\
g_{\alpha}(i) & =|| u_{\alpha}^{p-1}(i)|-| u^{p-1}(i)||, \\
y(i) & =|x(i)| .
\end{aligned}
$$

Clearly, $z_{\alpha}, w_{\alpha}, z, w \in l^{1}$ and $z_{\alpha}+z$ pointwise. By Lemma 2.1, we have

$$
\left|z_{\alpha}(i)\right| \leq w_{\alpha}(i) \text { for all } \alpha \in D \text {. }
$$

Also $\left\{g_{\alpha}\right\}$ is a bounded net in $\imath^{q}$ converging pointwise to $\theta$, where $\frac{1}{p}+\frac{1}{q}=1$. As $p>1$, this implies that $g_{\alpha} \stackrel{\omega}{\longrightarrow} \theta$. Moreover,

$$
\left\|w_{\alpha}-w\right\|=2^{p} p \sum_{i=1}^{\infty} g_{\alpha}(i) y(i)=2^{p} p\left\langle g_{\alpha}, y\right\rangle,
$$


where $\left\langle g_{\alpha}, y\right\rangle$ represents the value of the bounded linear functional $y \in l^{p}$ at $g_{\alpha} \in z^{q}$. An easy application of Theorem 2.2 to $(2.5)$ and (2.6) then gives the required result.

REMARK 2.5. Let $x$ be an element of $\imath^{p} \quad(1 \leq p<\infty)$ with finitely many nonzero coordinates. That $E(-x, x)$ is weakly closed can be verified easily. We do not know whether in Theorem 2.4 the bounded weak topology can be replaced by the weak topology or not.

COROLLARY 2.6. Let $M$ be a closed linear subspace of $i^{p}$ $(1<p<\infty), P_{M}$ the metric projection onto $M$. Then $P_{M}$ is continuous both from the strong to strong topology, and from the bounded weak to bounded weak topology on $Z^{P}$.

Proof. The uniform convexity of $z^{p} \quad(1<p<\infty)$ implies that $M$ is Chebyshev and $P_{M}$ is continuous from the strong to strong topology of $\imath^{p}$. To show that $P_{M}$ is continuous in the bounded weak topology of $\imath^{p}$, we first observe that for each $x \in X$, and for $M_{x}=[x], M_{x}^{\theta}$ is bounded weakly closed on account of Theorems 1.6 and 2.4. By the kernel intersection theorem of [4] we have $M^{\theta}=\bigcap_{x \in M} M_{x}^{\theta}$. Thus $M^{\theta}$ is bounded weakly closed. The result then follows from the following result of Holmes [3, p. 170]. If $M$ is reflexive, then $P_{M}$ is bw-continuous if and only if $M^{\theta}$ is bw-closed.

REMARK 2.7. The above has been essentially observed by Holmes [2] by using the fact that $l^{P}$ spaces $(1<p<\infty)$ have a weakly continuous duality mapping.

In the case of $l^{1}$, since strong and weak sequential convergence coincide, $E(-x, x)$ is weakly sequentially closed for each $x$. However, this property of $Z^{p}$ spaces is not present in $L^{p}(\mu)$ spaces $(1<p<\infty$, $p \neq 2$ ) where $\mu$ is a separable nonatomic measure. Lambert [9] has shown that $M^{\theta}$ is weakly sequentially dense for any finite dimensional Chebyshev 
subspace $M$ and consequently $E(-x, x)$ cannot be weakly sequentially closed for any $x$ in such spaces. In the following we show that $c_{0}$ also does not have this property.

THEOREM 2.8. Let $x$ be any point of $c_{0}$. Then $E(-x, x)$ is not weakly sequentially closed.

Proof. Let $x=\left(x_{1}, x_{2}, x_{3}, \ldots\right) \in c_{0}$. Take

$$
z_{n}(i)= \begin{cases}0 & , \text { if } i \neq n, \\ 2\|x\| \operatorname{sgn} x_{n}+x_{n}, & \text { if } i=n \text { and } x_{n} \neq 0, \\ 2\|x\| & , \text { if } i=n \text { and } x_{n}=0 .\end{cases}
$$

Then $\left\|z_{n}-x\right\|=\left\|z_{n}-2 x\right\|=2\|x\|$ for sufficiently large $n$. Hence $z_{n} \in E(x, 2 x)$ eventually. But $z_{n}$ converges weakly to $\theta$ and $\theta \notin E(x, 2 x)$. Therefore $E(x, 2 x)$ and consequently $E(-x, x)$ is not weakly sequentially closed.

COROLLARY 2.9. No one-dimensional Chebyshev subspace of. $c_{0}$ can have a weakly sequentially continuous metric projection.

Proof. Let $M=[x]$ be Chebyshev and $z_{n}$ be the sequence described in Theorem 2.8. Then $P_{M}\left(z_{n}\right) \in\{t x: 1 \leq t \leq 2\}$ for sufficiently large $n$, and $P_{M}(\theta)=\theta$. So $P_{M}\left(z_{n}\right)+\theta$. Hence $P_{M}$ is not weakly sequentially continuous.

\section{References}

[1] Mahlon M. Day, "Some characterizations of inner-product spaces", Trans. Amer. Math. Soc. 62 (1947), 320-337.

[2] R.B. Holmes, "On the continuity of best approximation operators", Symposizm on infinite dimensional theory, 137-157 (Annals of Mathematics Studies, 69. Princeton University Press and University of Tokyo Press, Princeton, New Jersey, 1972). 
[3] Richard B. Holmes, A course on optimization and best approximation (Lecture Notes in Mathematics, 257. Springer-Verlag, Berlin, Heidelberg, New York, 1972).

[4] Richard Holmes and Bernard Kripke, "Smoothness of approximation", Michigan Math. J. 15 (1968), 225-248.

[5] R.C. James, "Orthogonality in normed linear spaces", Duke Math. J. 12 (1945), 291-302.

[6] G.K. Kalisch and E.G. Straus, "On the determination of points in a Banach space by their distances from the points of a given set", An. Acad. Brasil. Ci. 29 (1957), 501-519.

[7] Victor Klee, "Convexity of Chebyshev sets", Math. Ann. 142 (1961), 292-304.

[8] Clifford A. Kottman and Bor-Luh Lin, "The weak continuity of metric projections", Michigan Math. J. 17 (1970), 401-404.

[9] Joseph Michael Lambert, "The weak sequential continuity of the metric projection in $L_{p}$ spaces", (Dissertation, Purdue University, 1970).

Department of Mathematics,

Indian Institute of Technology Kanpur,

Kanpur,

India. 